\title{
Efforts to eradicate obstetrical fistulas in the developing world: a personal journey
}

\author{
Rene Genadry, MD ${ }^{1}$
}

Key Words: obstetrical fistula, developing countries, health services, voluntary workers

\section{Introduction}

My work with international health has been primarily focused on obstetrical fistulas. It all started in 2004 with a call to Bob Wheeless, a colleague whom I have known since my early days at Hopkins, to ask him to participate in the Houston Everett course that year. While accepting gracefully and enthusiastically, he promptly related to me his travel activities since recently retiring. He had been approached by a group involved in humanitarian work in Niger to help care for poor and unfortunate women with obstetrical fistulas at the Niamey National Hospital. His exact words to me were, "We could use you if you are interested in that kind of surgery. We also had a difficult time communicating in French." For me it was the right time to get involved with international humanitarian work and for
"An unspeakable scent of decay, putrefaction, and something else for which words remain to be invented reached our nostrils. I saw no point in holding my breath or pinching my nose because the foulness invaded instantly, coloring our insides like a drop of India ink in a cup of water. In a way that children understand their own, we knew her to be innocent of her terrible, overpowering odor. It was of her, but it wasn't hers. Worse than the odor (since she must have lived with it for more than a few days) was to see her face in the knowledge of how it repulsed and revolted others. "

Abraham Varghese's 'Cutting for Stone' in

the right reasons. I had no idea the impact it will have on my future activities. The process is indeed addictive: I had seen such effects first-hand on the late Tom Elkins, whose untimely death in 1998 had left our plans for international work and programs utterly frustrated. When relating his work in West Africa, Tom's enthusiasm was indeed always evident and readily contagious.

Abdoulaye was our man in Niamey, a wonderful human being with outstanding surgical skills and even more diplomatic ones to

${ }^{1}$ The Department of Obstetrics and Gynecology, The Carver College of Medicine, The University of lowa, lowa City, lowa, USA

Please cite this paper as: Genadry, Rene. Efforts to eradicate obstetrical fistulas in the developing world: a personal journey. Proc Obstet Gynecol. 2012;2(4):Article 41 [ 7 p.]. Available from: http://ir.uiowa.edu/pog/.Free full text article.

Corresponding author: Rene Genadry, Department of Obstetrics and Gynecology, University of lowa, 51233 PFP, 200 Hawkins Drive, lowa City, IA 52242. Telephone (319)356-2306. rene-genadry@uiowa.edu.

This is an Open Access article distributed under the terms of the Creative Commons Attribution 3.0 Unported License (http://creativecommons.org/licenses/by/3.0), which permits unrestricted use, distribution, and reproduction in any medium, provided the original work is properly cited. 
manage a courtyard full of patients awaiting someone to accept to care for them. The problem of obstetrical fistula is a problem of utter poverty mixed with illiteracy and a lack of economic means or access to proper health care, if any. A more or less recent traumatic experience that ended in a stillbirth, and that led to the fistula leaving the woman dripping urine all day long rendering her "unclean" and almost leper-like vis-à-vis friends, family and village elders, added insult to injury. With no means of survival and a high rate of depression that often leads to suicide, lucky are the ones that find refuge in the hospital courtyard where a literal "fistula colony" fends for itself and its own. Many of these women walk from the bush to the capital because either they are too poor to afford transportation or transportation is denied them because the women constantly reek of urine. There, the women would sometimes wait weeks to months to years even for surgery; with no insurance or welfare, very few surgeons give preference to non-paying patients who customarily need to pay for their own medication and suture material let alone anesthesia, surgeon's fee and hospital bed. While awaiting a benevolent surgeon, many lived in the hospital courtyard, which is an outdoor space: the hospital does not officially provide them with shelter; the women just have nowhere else to go.

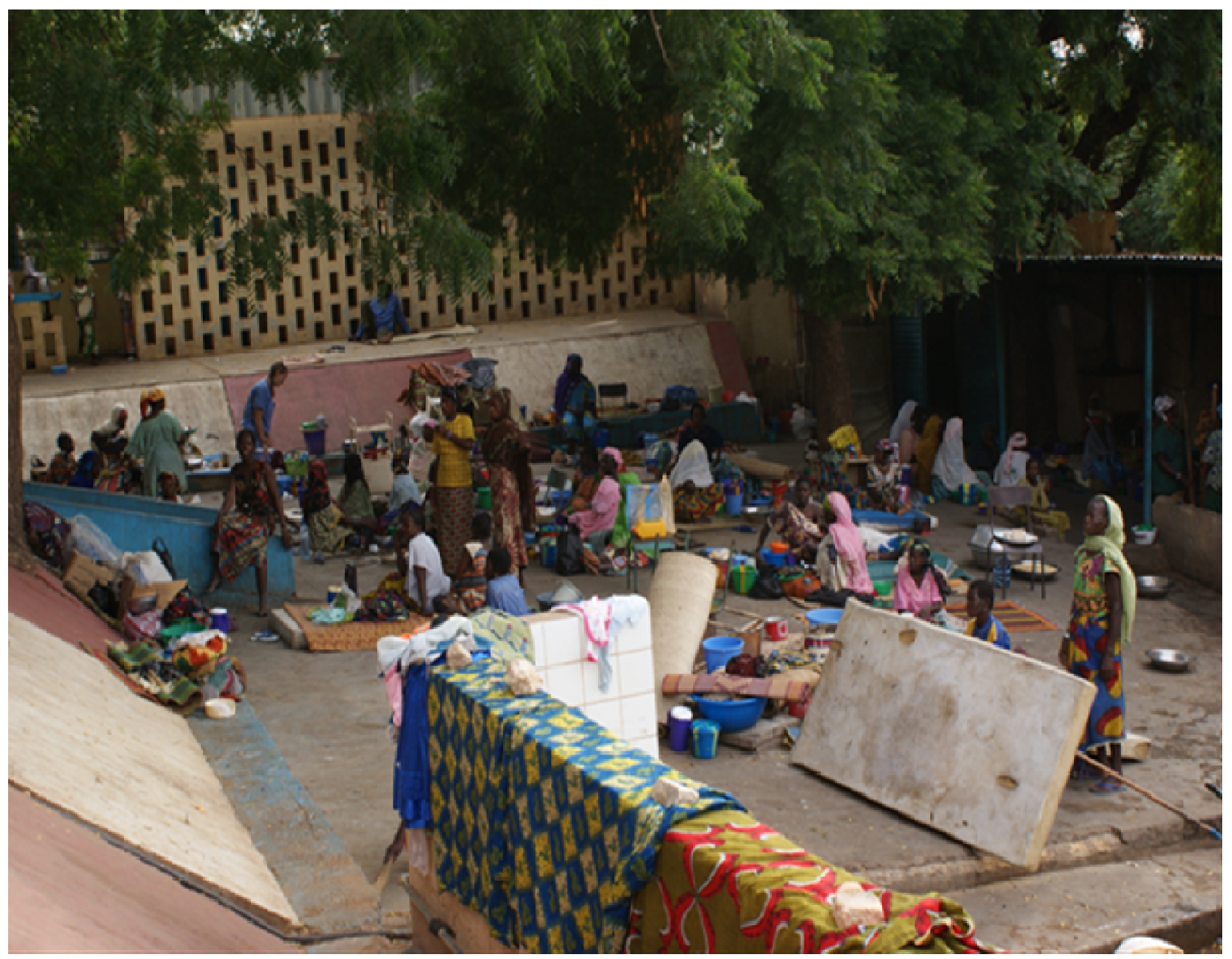

"The courtyard" 
Thus, Abdoulaye was their hero, with the necessary skill and willingness to perform surgery when time in the operating room permits. He found a welcome relief in a group of American doctors coming to help and complement the hospital while also bringing donated supplies to be used and to be left afterwards for use by anyone needing them. We had met in Paris for the flight to Niamey, capital of the state of Niger. Niger is considered the poorest country in Africa with the added distinction of being one of the poorest in the world. Barbara Margolies, an author and a teacher sensitized by her experience when working in Niger, had started organizing humanitarian missions to assist in a variety of ways with the goal of improving the lot of the people for whom she cared so much, including education, medical care, donations and a number of exchanges. Her local contacts facilitated the logistics of our travel and stay enormously. We had to plan effectively to catch the then only Air France flight of the week going into Niamey and the weekly flight coming out of there in order to be back to resume our usual daily activities. Abdoulaye had already made the necessary arrangements to inform potential patients of the pending arrival of the mission doctors through social and health care networks, radio announcements and the "courtyard" residents. There, as for any calamity, "word of mouth" carries loud and far, particularly when falling on receptive ears. The group that for the most part had never met before was made up of like-minded people with a variety of experiences in the fields of Medicine and Surgery, including Peace Corps volunteers and residents attracted by the promised richness of the experience. And it was a rich experience, indeed, as related in the following excerpt of a letter I sent Barbara after my first trip in 2004:

"... this a most wonderful experience... real benefit for those that are giving as well as those on the receiving end...And all things considered, more for the giver than the receiver!

...This has been the most fulfilling experience yet. Taking kids to camps and vacation, visiting needy people in various conditions, teaching folks to care for themselves and theirs are worthwhile endeavors and nothing gets lost. But improving a single person's life to such magnitude is so fulfilling that nothing comes close to describing the joy that elates both parties...

One gets the impression that what one is doing is but a drop in the ocean of misery, and resources could be better spent on prevention, education and more essential care. But in the meantime one realizes that there are human beings that are suffering emotionally and physically from lack thereof and need a helping hand today and now..." 
Needless to say, the flight back to Paris held a transformed group of caring individuals now in utter revolt against the inequities and misery the world offers, while those they left behind live it all the time....and with a smile at times.

Since my first trip, I have participated in missions to Niger and assessment missions to Benin, Mali, Ethiopia, Nigeria and Bangladesh. During one of these trips, I was introduced to a little baby girl with bladder exstrophy. She had been in the hospital in Niamey for a few months, with no one able to fix her congenital abnormality that kept her constantly leaking urine through the bladder opening into the lower abdomen, exposing her to the risk of infections and renal compromise. Through the generous and benevolent assistance of Ronald Peterson, president of the $\mathrm{JHH}$, and the expert and caring contribution of John Gearhart, a prominent pediatric urologist with interest in the treatment of exstrophy of the bladder, she was hospitalized and treated at Hopkins. Today she is enjoying a life that would have otherwise been denied to her had she not been lucky enough to find caring and giving individuals and institution.

\section{Challenges}

The first mission in Niger was a humbling experience. We had brought with us donated equipment to be left after our departure and the necessary material to properly evaluate, operate when needed and provide care as needed. Taking a history from patients proved quite challenging. At times, two or even three individuals were needed to communicate the information eventually in Hausa, and finally in
English, as told in her particular dialect by the individual patient. For every patient, it became critical to confirm such related information by additional evaluation, particularly before surgical correction. We had to operate with limited resources despite our modern equipment: with frequent power losses, operating with flashlights, and for the more seasoned and luxuriously inclined, with battery-operated headlights. Nonetheless the frustration and the heat added to the technical challenges encountered in patients that had undergone a number of previous surgical procedures. Working under those circumstances made us realize what hardships our hosts, patients and health care workers had to tolerate and overcome in order to care for their own. To see how people had to cope with conditions and circumstances that were hard to accept by any standard and yet survive with a smile not only gives a sense of the resilience of the culture but also a deep sense of shame and guilt at being so privileged in that respect. To experience the ingenuity of the people dealing so positively with adversity was a lesson in adaptability. To realize the magnitude of the task of eradicating obstetric fistulas as was done in the developed world was indeed overwhelming. Help was needed at all levels and in all directions. Fortunately, the process was already under way as people in positions of responsibility had been tackling the issues with a similar sense of ineptitude. One has to do what is in one's means to contribute to some solution for this unacceptable scourge.

The obstetrical fistula is the end result of a dysfunction that began at least nine months earlier. Lacking the economical and logistical means as well as the 
knowledge, availability and accessibility of prenatal care, these women are the survivors of a population that contributes to the high maternal mortality in those countries sharing similar handicaps. But such survival comes at a very high price: loss of offspring, loss of quality of life, loss of dignity. Too many factors must be addressed if solutions are to be found. For example, too little systematic knowledge is available. Though a growing number of humanitarians have been trying to assist this patient population, more evidence-based information and effective cooperation are needed.

\section{Collaboration}

As has already occurred in the industrialized world, in order to eliminate the problem altogether, a collaborative effort that will lead to a multipronged approach to resolve all the issues must be started. An exhaustive literature review demonstrated the absence of reliable data regarding the prevalence of obstetrical fistulas in the world. There was also a remarkable paucity of clinical data that could be pooled together to understand the current state of the care and cure of obstetrical fistula. With the generous help and supportive guidance of Dr. Amy Tsui, director of the Gates Institute at the Johns Hopkins Bloomberg School of Public Health (JHSPH), a meeting was organized to bring together such a group of investigators, researchers, health care and social workers as well as government representatives to help define the research needs and priorities. This meeting was held in July 2005 at the JHSPH in Baltimore, Maryland under the combined WHO-UNFPAFIGO sponsorship.
The objectives of the meeting were to gather as many of the available stakeholders as could be assembled in order to consider all these related issues and challenges to the advancement of the scientific knowledge of the risks and treatments of obstetric fistula. The immediate goals were 1) to prioritize research questions regarding public health and clinical responses to obstetric fistula, including informing the design of a study addressing one or more key research needs, and 2) to inform the public health response to and management of obstetric fistula. A group of over seventy clinicians and public health experts from the United States, Canada, the United Kingdom, and countries in Africa, Asia, and Latin America grappled with the incidence and prevalence of obstetric fistula and the best ways to address this public health problem. This led to the publication of a supplement to the IJOG on the same topic (IJOG Volume 99 suppl. 1, Nov 2007.)

The Editor's note was appropriately titled: "Dead Women Walking" and included the following comment: "The data presented in this IJGO supplement emphasize that virtually nothing about the diagnosis of fistula, the treatment of fistula, or the outcome of fistula repair is standardized, and that no evidencebased relationships have been established between diagnosis, treatment, and outcome."

In this supplement, the need for reliable and valid data was repeatedly emphasized and readily acknowledged. However, to date a staging system has not yet been established, mainly because each author defines success differently. The need for a standard 
classification and a case-reporting system was also stressed in the supplement. Proper actions in the areas of research, programming and advocacy could hardly be sustained without the availability of such data.

Therapeutic strategies should take into account the patient in her totality: the multifaceted aspects surrounding the issues of obstetrical fistula require the partnership between the public health and clinical domains. Sharing knowledge and resources is critical to collect evidence-based data and develop strategies to improve the patient's physical condition as well as her well-being (emotional, psychological, and economical) and overall quality of life.

Most importantly, preventive strategies at all levels should be the cornerstone of any program aimed at eradicating obstetrical fistula. Only with the partnering of the central governmental and local communal bodies could this be provided and include the needed resources at all levels of the prevention strategy. Prevention is indeed the key to ending obstetrical fistulas. Prolonged, obstructed labor occurs most frequently in countries where early marriage and early childbearing (before the pelvis is fully developed), malnutrition and small stature, poor general health and poverty are commonplace; where access to emergency obstetrical care is limited or unavailable; and where multiparity is valued and encouraged. Communitylevel prevention measures and social support mechanisms can help reduce the burden of disease. In the absence of empirical evidence to guide prevention and treatment, the ability to develop effective fistula eradication programs is significantly compromised: research on the factors that contribute to the risk of developing an obstetrical fistula, such as lack of education, early pregnancy and severe poverty, is necessary if they are to be prevented.

\section{Research Priorities}

Research priorities were identified in six main areas: (A) assess the burden of disease attributable to obstetrical fistula; (B) identify effective community-level prevention measures such as delaying early childbearing, raising awareness on the need for skilled attendance at birth, and mobilizing response to signs of pregnancy complications; (C) develop a standard classification system and establish an international registry of fistula cases; (D) conduct operations research on barriers to emergency obstetric care at the community, facility, provider, and patient levels; (E) identify evidence-based practices for successful fistula management, including catheterization duration and how to handle residual stress incontinence, diversion protocols, and repeat fistula repair; and $(F)$ assess the low-cost methods of treating obstetrical fistulas in resource-constrained settings.

In the meantime, adequate medical care and emergency obstetric services, particularly cesarean deliveries, could be made available through strong educational efforts from communities and public health professionals. For example, we may be able to reduce the number of pregnancies at an early maternal age by providing education regarding the risk of childbearing before reproductive maturity is reached, along with delaying marriage and/or providing education in family planning. As part of 
the overall care, counseling for psychological and emotional damage, and support for social reintegration, should be provided in addition to surgery.

The task is huge and requires concerted and collaborative efforts from a variety of entities to advance the cause of these wretched women and whose only misfortune is to be in the wrong place at the wrong time. Work has to be done on both time and place: time is money and places are peopled. The two most critical ingredients, committed people and availability of resources, are critical to the eradication of this condition whose existence remains "a shameful affront to all those concerned with and responsible for the constant improvement of public health."

\section{Future Directions}

A number of subsequent meetings led to the development of a proposal for a prospective multicountry study to assess the quality of life before and after surgery and to collect standardized clinical data to define prognostic factors. These would allow for assessment of the numerous non-validated personal classifications regarding their prognostic value, and may lead to the adoption of an international classification to facilitate data collation and comparison. The economic downturn significantly delayed the implementation of the study due to unavailability of promised funds. However, some funds started trickling in through the efforts of committed people at the UNFPA who ensured that the study got under way. Study is in progress in Ethiopia and Niger while it has been completed in Bangladesh.

Opportunities for international work are available on many levels. Interestingly, one does not necessarily have to travel to effectively contribute to improving the lot of those in need. Whatever the ability and interest of each one of us, there is a need that one can fulfill. The key is to tap at the proper door for it to open a wide range of possibilities from humanitarian and non-medical options to active participation in overseas medical programs. It is my hope to develop a venue for physicians interested in learning more about the issues related to fistulas, and in teaching the updates in operative and perioperative care of patients with pelvic floor abnormalities. Thus, local physicians and those visiting with them can exchange evidence-based information in their respective fields of endeavor to improve the care provided individually and to the individual patient. This should ultimately result in a repository of shared information for the betterment of the care of the patients and the education of the caregivers. The development of a research infrastructure will produce answers to the most urgent questions in relation to care and prevention of these abnormalities. 